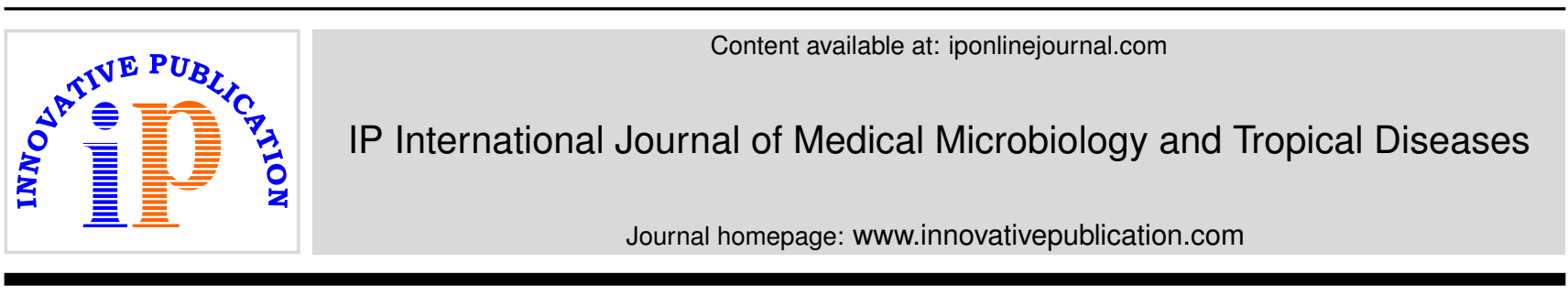

Original Research Article

\title{
Antimicrobial susceptibility profile of Staphylococcus aureus isolated from pyogenic infections-Variations encountered at secondary and tertiary care level centres
}

\author{
Mandeep Sen ${ }^{1}$, Sana Islahi ${ }^{1, *}$, Anupam Das' ${ }^{1}$, Jyotsna Agarwal $^{1}$ \\ ${ }^{1}$ Dept. of Microbiology, Dr. Ram Manohar Lohia Institute of Medical Sciences, Lucknow, Uttar Pradesh, India
}

\section{A R T I C L E I N F O}

\section{Article history:}

Received 24-10-2019

Accepted 15-11-2019

Available online 11-01-2020

Keywords:

Pyogenic Infections

Secondary and Tertiary Health Care

Level Centre

Antimicrobial Resistance

\begin{abstract}
A B S T R A C T
Introduction: The bacterial profile of pus samples in many studies remain the same, but the antibiotic resistance pattern of these isolates has shown a lot of variations.

Objective: Comparative study of bacteriological profile and antimicrobial susceptibility of Staphylococcus aureus isolated from pus and wound swab of patients at secondary and tertiary care level centres.

Material and Methods: This observational retrospective study was conducted at the department of Microbiology in a super specialty Post Graduate institute. Antibiotic sensitivity testing of Staphylococcal isolates was performed by modified Kirby Bauer's disc diffusion method as per CLSI 2017 and EUCAST guidelines. Automated susceptibility testing was performed by Vitek-2 Compact system.

Conclusions: This study provides a comparative All medical pus specimens from both institution as well as from SRCLI (State Referral Centre for Clinical Lab Investigation) were included of Staphylococcus aureus isolated from pus and wound swab of patients to formulate the local antibiotic policy to initiate the appropriate empirical antibiotic treatment at secondary and tertiary care level centres.

Results: Out of 997(59.38\%) samples showing single growth, Gram positive organisms were 216 (35.23\%) for institutional and 195 (50.78\%) for SRCLI (State Referral Centre for Clinical Lab Investigation). 85\% and $91 \%$ isolates of Staphylococcus aureus were resistant to penicillin followed by erythromyc in $(53.5 \%$ and $49 \%)$ and cefoperazone-sulbactum (28.5\% and $14 \%)$, while $31(55 \%)$ and $36(34.61 \%)$ isolates were MRSA respectively for institutional and SRCLI. Even the higher antibiotic like linezolid showed 2.5\% resistance $(\mathrm{P}=0.5054)$ in MRSA isolates of SRCLI compared to $0 \%$ in institutional samples, which was, though not statistically significant, but is of great concern. Vancomycin showed no resistance in both areas.

(C) 2019 Published by Innovative Publication. This is an open access article under the CC BY-NC-ND license (https://creativecommons.org/licenses/by/4.0/)
\end{abstract}

\section{Introduction}

Wound infection can be caused by variety of organisms which may co-exist as polymicrobial communities. Although, the bacterial profile of pus samples in many studies remain the same, the antibiotic resistance pattern of these isolates has shown a lot of variations. Staphylococcus aureus is a Gram positive cocci, which is a part of normal flora occupies anterior nares, nasopharynx, perineal area, skin and colonizer of mucosa. S.aureus is one of the emerging pathogen in hospital settings and c

\footnotetext{
* Corresponding author.

E-mail address: islahi.sana@gmail.com (S. Islahi).
}

ommunity settings worldwide. ${ }^{1}$ It can cause variety of infections including skin infections, joint infections, urinary infections, to pneumonia to septicaemia. ${ }^{2}$ Methicillin Resistant Staphylococcus aureus (MRSA) is an emerging multidrug resistant bacteria worldwide, prevalence $\mathrm{r}$ anging from $4.6 \%$ to $54.4 \% .^{3-6}$ MRSA grouped under HA MRSA (Healthcare associated methicillin resistant Staphylococcus aureus) and CA MRSA (Community acquired methicillin resistant Staphylococcus aureus). HA MRSA is a pathogen usually acquired during prolonged or frequent hospitalizations and CA MRSA usually affects healthy people, transmission of pathogen occur within community. MRSA is also accountable for outbreak 
in many countries including USA, Europe, Australia, Japan. $^{7}$ MRSA transmission occurs in hospitals through infected patients or staff or can be spread by patients or staff colonized MRSA. ${ }^{8}$ mecR 1 and mecI gene which is responsible for production of penicillin binding protein which has low affinity to beta lactam antibiotics is one of the mechanism explained for MRSA. ${ }^{9,10}$ Knowledge regarding prevalence of MRSA is important to know for choosing empiric therapy and to screen colonized patients or staff. As Staphylococcus aureus is most common among pus samples, we have selected to do this study in this region. The aim of the present study is to compare the bacteriological profile and antimicrobial susceptibility of Staphylococcus aureus isolated from pus and wound swab of patients at secondary and tertiary care level centres. The present study is unique of its kind as to the best of our knowledge there is no similar comparative published study in the past.

\subsection{Procedure}

\subsection{Study design}

An observational retrospective study was conducted from January 2017 to December 2017 at the Microbiology Department of the Post-Graduate Super Specialty Institute. This study was carried out on all medical pus specimens from different study population anatomical sites which were both institutional as well as from SRCLI (State Referral Centre for Clinical Lab Investigation) which includes samples referred from twelve secondary care level health care centres. As per CLSI (Clinical and Laboratory Standards Institute) 2017 and EUCAST (European Committee on Antimicrobial Susceptibility Testing) guidelines, the antibiotic sensitivity testing of Staphylococcal isolates were carried out using the modified Kirby Bauer disc diffusion process. The Vitek-2 Compact system performed automated susceptibility analysis. All pus specimens are immediately inoculated on Blood agar, MacConkey agar, Brain Heart Infusion Broth after obtaining them at the department of Microbiology. All media plates are incubated at $37^{\circ} \mathrm{C}$ for 24 hours after inoculation. During processing the samples, sterile precautions were taken. Identification of isolates from positive culture was done as per standard tests for identification, by Gram staining, motility and biochemical tests like catalase, coagulase, indole, methyl red, Voges-Proskauer, citrate, urease, phenyl pyruvic acid test and oxidase test.

\subsection{Antibiotic sensitivity testing}

Antibiotic sensitivity testing of Staphylococcal isolates was performed by modified Kirby Bauer's disc diffusion method on Mueller Hinton agar as per CLSI 2017 and EUCAST guidelines. Automated susceptibility testing was performed by Vitek-2 Compact system. Standard antibiotics like, cefazoline $(30 \mathrm{mcg})$, penicillin (2 U), cotrimoxazole $(25 \mathrm{mcg})$, cefoxitin $(30 \mathrm{mcg})$, clindamycin (2mcg), erythromycin (15mcg), linezolid (30 mcg) and vancomycin $(30 \mathrm{mcg})$ were tested. All the culture media, biochemical media and antibiotic discs used were obtained from Hi Media.

\subsection{Ethics}

All applicable institutional guidelines for the participants were followed. It was entirely an observational study. Confidentiality of identity was ensured for all the persons and a verbal consent was obtained prior to filling up of the questionnaire. This retrospective study was approved by Institutional ethical committee.

\subsection{Statistical Analysis}

The statistical analyses were performed using statistical package for social science software (SPSS version 16.0) and Microsoft office excel 2010. A p-value of $<0.05$ was considered statistically significant.

\section{Results}

Microbiology department obtained a total of 1679 samples and tested for aerobic culture and sensitivity; out of which 997 (59.38\%) showed single growth. Of which 613 were institutional and 384 were of district hospitals through SRCLI. Isolation of Gram positive (Table 1) and Gram negative organisms were 216(35.23\%) and $397(64.77 \%$ ) respectively for institutional and $195(50.78 \%)$ and $189(49.22 \%)$ for SRCLI. Antibiotic susceptibility screening for the identification of MRSA and antibiotic resistance trends for institutional and SRCLI was performed on 75 and 104 Staphylococcus aureus isolates respectively (Table 2). $91 \%$ and $81 \%$ isolates were resistant to Penicillin followed by erythromycin (64\% and 49\%) and gentamicin (54.6\% and $51 \%$ ), resistance to linezolid was observed in 0 and $3 \%$ cases while $41(55 \%)$ and $36(34.61 \%)$ isolates were MRSA respectively for institutional and SRCLI. Vancomycin showed $0 \%$ resistance in both. (Table 3)

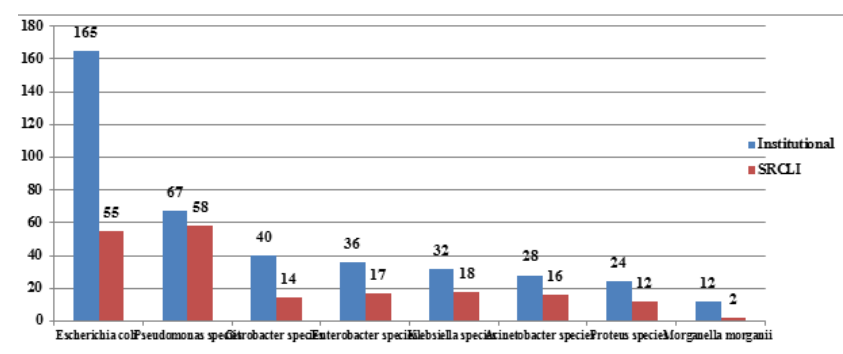

Fig. 1: Gram negative isolates from pus and wound swab samples 
Table 1: Gram positive isolates from pus and wound swab samples

\begin{tabular}{llllll}
\hline Isolates & OPD & IPD & ICU & Institutional & SRCLI \\
Enterococcus species & 38 & 8 & 3 & $49(22.68 \%)$ & $14(7.2 \%)$ \\
Staphylococcus aureus & 69 & 5 & 1 & $75(34.72 \%)$ & $104(53.3 \%)(\mathrm{P}=0.0176)$ \\
$\begin{array}{l}\text { Coagulase Negative Staphylococci } \\
\text { (CoNS ) }\end{array}$ & 67 & 20 & 4 & $91(42.12 \%)$ & $76(38.9 \%)$ \\
$\begin{array}{l}\text { Streptococcus pyogens } \\
\text { Total }\end{array}$ & 1 & 0 & 0 & $1(0.4 \%)$ & $1(0.5 \%)$ \\
\hline
\end{tabular}

Table 2: Antibiotic resistance pattern in Staphylococcus aureus from pus and wound swab samples

\begin{tabular}{lllll}
\hline Antibiotics & \% Resistant strain & & & \\
OPD (n=69) & IPD (n=5) & ICU (n=1) & SRCLI (n=104) \\
Penicillin & 90 & 80 & 100 & 81 \\
Gentamycin & 54 & 60 & 100 & 51 \\
Tetracycline & 38 & 40 & 100 & 24 \\
Doxycycline & 14 & 20 & 100 & 49 \\
Erythromycin & 67 & 40 & 0 & 3 \\
Linezolid & 0 & 0 & 0 & 19 \\
Clindamycin & 22 & 40 & 0 & 0 \\
Vancomycin & 0 & 0 & 0 & 34.6 \\
Cefoxitin & 53 & 60 & 100 & \\
\hline
\end{tabular}

Table 3: Sensitivity pattern of antibiotics to MRSA from pus and wound swab samples

\begin{tabular}{llll}
\hline Antibiotics & \% Resistant strain in MRSA & SRCLI (n=36) \\
& OPD $(\mathbf{n = 3 6})$ & IPD $(\mathbf{n = 3})$ & 100 \\
Penicillin & 100 & 100 & 75.6 \\
Erythromycin & 86 & 66.6 & $71.05(\mathrm{P}=0.3417)$ \\
Tetracycline & 50 & 66.6 & 25.7 \\
Clindamycin & 31.25 & 66.6 & $71.05(\mathrm{P}=0.0041)$ \\
Gentamycin & 16.5 & 66.6 & $15.3(\mathrm{P}=0.1961)$ \\
Doxycycline & 5 & 33.3 & $2.5(\mathrm{P}=0.5054)$ \\
Linezolid & 0 & 0 & 0 \\
Vancomycin & 0 & 0 & \\
\hline
\end{tabular}

\section{Discussion}

Staphylococcus aureus is one of the most prominent organisms causing skin or soft tissue abscesses. In addition to form pus it is also responsible for the pneumonia, toxic-shock syndrome, exfoliative skin disease, and enteritis. S.aureus colonize the human skin, nails, and nares and disseminate via physical contact and aerosols. Staphylococcal invasion is generally caused due to breaches in local defense mechanism, like skin cuts or hair follicle trauma, which manifests as abscess formation and severe inflammation of surrounding tissues. Improper use of antibiotics over the past few decades has led to drugresistant strains, designated MRSA (methicillin-resistant $S$. aureus).

In our study out of 1679 samples, 997(59.38\%) were culture positive which was found close to the study done by Shrestha et al. ${ }^{11}(50 \%)$. However, higher rate were also reported, $(78.55 \%)$ by Bankar et al. ${ }^{12}$ and $(71.49 \%)$ by Nithya et al. ${ }^{13}$
In our study Gram positive and Gram negative organisms isolated were $35.23 \%$ and $39.7 \%$ respectively for institutional isolates. There was a preponderance of Gram negative organisms observed in our study. This was in accordance with the study by Nithya et al. ${ }^{13}$ while Bhatta et al. ${ }^{14} \&$ Shrestha et al. ${ }^{11}$ reported Staphylococcus aureus to be the most prevalent bacteria isolated from the cases of wound infections which was similar to the our study with isolates of SRCLI which was found to be statistically significant $(\mathrm{P}=0.0176)$.

Different studies have been performed to assess the bacterial profile and the antibiotic susceptibility pattern in pus samples. This is particularly relevant for the treating physician who needs to start empirical treatment of patient until the lab culture reports are awaited. ${ }^{15}$

We have performed the antibiotic susceptibility testing on Staphylococcus aureus to see the MRSA and resistance pattern of MRSA to start the empirical treatment before the culture reports are available. In our study it was observed that $53 \%-60 \%$ isolates were MRSA which was similar to 
the MRSA rate observed by Pushpalatha et al. $(53.96 \%)^{16}$ and Arti Tyagi et al. ${ }^{17}$ who did a large study on 2,080 pus samples at AIIMS, New Delhi, documented that the MRSA prevalence rate was $44 \%$ of all $S$. aureus isolates, while MRSA in SRCLI isolates in our study were lower (36.4\%) than these studies. The prevalence of MRSA in our study was higher than the study by Subedi and Brahmadathan. ${ }^{18}$ All isolates were sensitive to vancomycin, rifampicin and teicoplanin.

MRSA was predominantly noticed in males and 1545 years age group in this study, which is supported by Dechen $\mathrm{C}$ Tsering et al. ${ }^{19}$ Geyid A et al. ${ }^{20}$ who documented that gender and age are not risk factor for the acquisition or colonization of MRSA. Prevalence of MRSA varies in different countries. Over 50\% prevalence rate of MRSA was reported in Portugal and Italy; $25 \%$ in England, Greece and France; 2\% in the Netherlands and Switzerland. ${ }^{21}$ Prevalence of MRSA ranged from $23.6 \%$ in Australia to over $61 \%$ in Taiwan and Singapore, and more than $70 \%$ in Japan and Hong Kong. ${ }^{2}$

The most peculiar feature of our study was the variation in the antibiotic susceptibility of MRSA between secondary and tertiary care centre which is of great concern. In our study it was found that SRCLI isolates with MRSA were found to be more resistant to antibiotics like tetracycline, gentami cin, doxycycline. Though variation in the antibiotic susceptibility of MRSA between secondary and tertiary care centre for tetracycline $(\mathrm{P}=0.3417)$ and doxycycline $(\mathrm{P}=0.1961)$ were not statistically significant, but for gentamicin $(\mathrm{P}=0.0041)$, it was found to be statistically significant. Even the higher antibiotic like linezolid showed $2.5 \%$ resistance $(\mathrm{P}=0.5054)$ in MRSA isolates of SRCLI compared to $0 \%$ in institutional samples, which was, though not statistically significant, but must be notified. This was confirmed by distributing the multiple choice questionnaire to the doctors of the SRCLI centres. Most of them presented linezolid as their first choice in pus samples caused by Gram positive bacterias, leading to its higher resistance.

Difference in the antibiotic susceptibility of MRSA may be due to differences in the empirical treatment or lack of culture facilities in the remote areas. It is of great concern that in our study some strains showed resistance to higher antibiotics especially at secondary care level centres, so these drugs should be used very cautiously to prevent emergence of antibiotic resistance.

\section{Conclusions}

Higher resistance of certain antibiotics to MRSA was found at secondary level than at tertiary level care centres which is alarming, probably due to misuse of these antibiotics. This study provides a comparative antibiotic susceptibility of Staphylococcus aureus isolated from pus \& wound swab of patients at secondary and tertiary care level centres, to formulate the local antibiotic policy to start the appropriate empirical antibiotic treatment before the culture reports are available.

\section{Source of Funding}

None.

\section{Conflict of Interest}

None.

\section{References}

1. Francois P, Pittet D, Bento M, Pepey B, Vaudaux P, et al. Rapid detection of methicillin-resistant Staphylococcus aureus directly from sterile or none sterile clinical samples by a new molecular assay. $J$ Clin Microbiol. 2003;41(1):254-260.

2. Kaur H, Purwar S, Saini A, Kaur H, Karadesai SG, et al. Status of methicillin-resistant Staphylococcus aureus infections and evaluation of PVL producing strains in. JKIMSU. 2012;1(2):43-51.

3. Giacometti A, Cirion O, Schimizzi AM. Epidemiology and Microbiology of surgical wound infections. Clin Microbiol. 2000;38(2):918-922.

4. Swanston WH. Methicillin resistant Staphylococcus aureus. West Indian Med J. 1999;48(1):20-22.

5. Mcdonald M. The epidemiology of methicillin resistant Staphylococcus aureus: surgical relevance 20 years on. Aust NZ J Surg. 1997;67(10):682-685.

6. Kac G, Buu-Hoi A, Herrison E. Methicillin resistant staphylococcus aureus nosocomial acquisition and carrier state in a wound care centre. Arch Dermatol;136:735-739.

7. Ayliffe GA, Duckworth GJ, Brumfitt W. Guidelines for control epidemic methicillin-resistant Staphylococcus aureus. J Hasp infect. 1986;7(193):201.

8. Stefani S, Varaldo PE. Epidemiology of methicillin-resistant Staphylococci in Europe. Clin Microbiol Infect. 2003;9:1179-1186.

9. Anand KB, Agrawal P, Kumar S, Kapila K. Comparison of cefoxitin disc diffusion test, oxacillin screen agar, and PCR for mecA gene for detection of MRSA. Indian J Med Microbiol. 2009;27(1):27-29.

10. Hackbarth CJ, Chambers HF. Methicillin-resistant staphylococci: genetics and mechanisms of resistance. Antimicrob Agents Chemother. 1989;33:995-999.

11. Shrestha B, Basnet RB. Wound infection and antibiotic sensitivity pattern of bacterial isolates. Post-Graduate. Med J NAMS. 2009;9(1):1-6.

12. Bankar N, Wankhade A, Brahmane RB, Hathiwala R, Chandi DH. Bacteriological profile of pus/ wound swab and antimicrobial susceptibility of Staphylococcus aureus isolated from pus \& wound swab of indoor patients of tertiary care hospital. Int J Innov Res Med Sci. 2018;3(4):1976-1980.

13. Gomatheswari NS, Jeyamurugan T. Bacteriological profile and the antibiotic susceptibility pattern of microorganisms isolated from pus/wound swab isolates in patients attending a tertiary care hospital in South India. Int J Curr Microbiol App Sci. 2017;6(10):1405-1413.

14. Bhatta CP, Lakhey M. The distribution of pathogens causing wound infection and their antibiotic susceptibility pattern. J Nepal Health Res Council. 2007;5(1):22-25.

15. Rameshkannan S, Nileshraj G, Rameshprabu S, Mangaiarkkarasi A, Meherali R. Pattern of pathogens and their sensitivity isolated from pus culture reports in a tertiary care hospital puducherry. Indian J Basic Appl Med Res. 2014;4(1):243-248.

16. Hanumanthappa P, Vishalakshi B, Krishna S. A Study on aerobic Bacteriological profile and Drug sensitivity pattern of Pus samples in a tertiary care hospital. Int J Curr Microbiol App Sci. 2016;5(1):95-102.

17. Tyagi A, Kapil A, Singh P. Incidence of Methicillin Resistant Stahylococcus aureus (MRSA) in Pus Samples at a Tertiary Care Hospital. AIIMS. 2008;9(1):33-35. JIACM. 
18. Subedi S, Brahmadathan KN. Antimicrobial susceptibility patterns of clinical isolates of Staphylococcus aureus in Nepal. Clin Microbiol Infect. 2005;11(3):235-237.

19. Tsering DC, Pal R, Kar S. Methicillin - Resistant Staphylococcus aureus: Prevalence and Current Susceptibility Pattern in Sikkim. $J$ Glob Infect Dis. 2011;3(1):9-13.

20. Geyid A, Lemeneh Y. The incidence of methicillin-resistant strains of Staphylococcus aureus strains in clinical specimens in relation to their beta-lactamase producing and multiple-drug resistance properties in Addis Ababa. Ethiop Med J. 1991;29:149-161.

21. Orrett FA. Antimicrobial resistance in Trinidad: hospital practice strains verses community practice strains of Staphylococcus aureus. Jpn J Infect Dis. 1997;25:663-666.

22. Diekema DJ, Pfaller MA, Schmitz FJ, Smayevsky J, Bell J, et al. Survey of infections due to Staphylococcus species: frequency of occurrence and antimicrobial susceptibility of isolates collected in the United States, Canada, Latin America, Europe, and the Western Pacific region for the SENTRY Antimicrobial Surveillance Program. Clin Infect Dis. 1997;32(2):114-132.

\section{Author biography}

Mandeep Sen Professor

Sana Islahi Senior Resident

Anupam Das Associate Professor

Jyotsna Agarwal Professor and Head

Cite this article: Sen M, Islahi S, Das A, Agarwal J. Antimicrobial susceptibility profile of Staphylococcus aureus isolated from pyogenic infections-Variations encountered at secondary and tertiary care level centres. Int J Med Microbiol Trop Dis 2019;5(4):188-192. 